
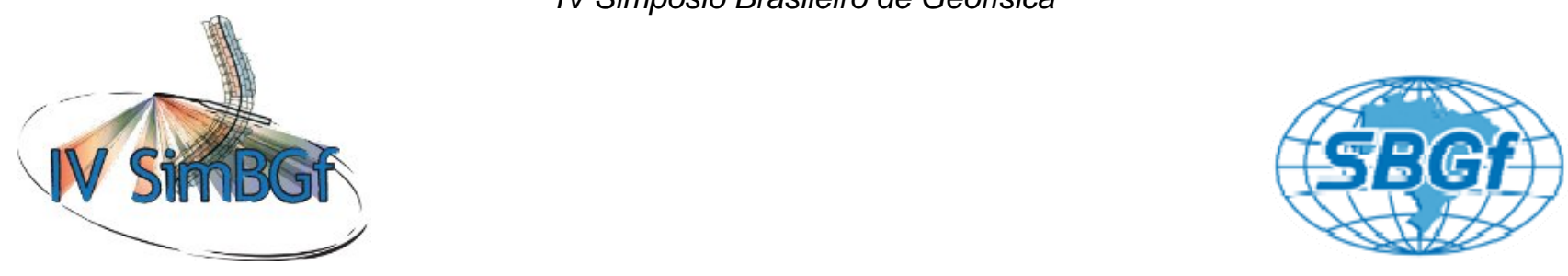

\title{
Viscoelasticidade aplicada a estudos de prospecção de petróleo
}

1Luiz Fernando da Silva, 2 Marcos Botelho

1,2- CPGG/Universidade Federal da Bahia

\section{Resumo}

Os estudos de modelagem são de grande relevância na investigação e prospecção de petróleo. Um dos processos de investigação do meio geológico é o da utilização de um modelo viscoelástico (Modelo de Maxwell, Kelvin-Voight, Solido Padrão, Burger etc) na implementação numérica da equação da onda e assim obter informações de subsuperfície (snapshots, sismogramas). Uma das vantagens de usar esses modelos é que eles incorporam a atenuação do meio neste processo de modelagem. Nosso projeto consiste em utilizar um modelo viscoelástico mais realístico (Modelo de Burger) e dessa forma recuperar informações do meio com mais exatidão. Inicialmente foi implementado o Modelo de Maxwell para estudos e testes de algoritmo. Os resultados apresentados neste trabalho são exclusivamente do modelo de Maxwell.

\section{INTRODUÇÃO}

A investigação de um meio sobre o qual se quer obter informação é realizada por meio de uma perturbação (uma onda gerada artificialmente) e então verificamos como o meio reagiu a essa perturbação. Essa análise do comportamento do meio pode ser por intermédio do campo de velocidades, do tensor das tensões e das deformações, do traço sísmico etc. Na modelagem via processamento de sinal, o traço sísmico apresenta uma grande relevãncia para geração e análise dos dados. No processo de modelagem através de modelos elásticos e viscoelásticos, as equações constitutivas e dinâmicas do meio é que exercem uma grande importância. O procedimento adotado para modelar é o de acoplamento das: equações constitutivas do meio (Lei de Hooke); Variáveis da Memória (equação constitutiva hereditária, memória evanescente, tensão e deformação em função do tempo, função Fluência e função Relaxação); Equação dinâmica do meio (equação de Euler, equação da onda), equação de Christofell etc. O objetivo principal do acoplamento dessas equações é descrever de maneira exata o comportamento do meio devido a uma perturbação. Os trabalhos publicados pelos pesquisadores dessa área ao longo dos anos e nos dias atuais, basicamente buscam melhorar esse acoplamento entre as equações para obter resultados mais precisos. Fatores tais como: dispersão e atenuação, são relevantes na pesquisa de modelagem e por isso têm-se muito interêsse na investigação desses fenômenos. Os mecanismos de atenuação e dispersão do meio são simulados através da parametrização do fator da qualidade Q modelado pelo, (no caso viscoelástico), modelo viscoelástico utilizado (por exemplo: modelo de Maxwell( uma mola e um amortecedor em série) ou modelo de Kelvin-Voight (uma mola e um amortecedor em paralelo, etc), das equações constitutivas (Lei de Hooke: que descreve as tensões e deformações sofridas pelo meio devido a essa perturbação),dos mecanismos de memória evanescente:que são descritos através da Equação Constitutiva Hereditária, onde as tensões são relacionadas no tempo com as deformações e as deformações são relacionadas no tempo com as tensões. Esse processo de convolução gera, respectivamente as funções relaxação e fluência. Ou seja, o processo segue alguns passos que são direcionados conforme o modelo elástico ou viscoelástico adotado; então prossegue-se com os cálculos para encontrarmos as equações que regem o meio através do 
modelo adotado e depois segue com uma implementação por um método numérico.

Nossa pesquisa bibliográfica foi feita em livros e trabalhos publicados por vários pesquisadores e em varias áreas do conhecimento humano. Analisando o estado da arte percebemos que existe ainda uma série de questões ainda a serem resolvidas. Nossa pesquisa pretende utilizar o modelo de 04 parametros ( Modelo de Burger) no processo de modelagem. $O$ modelo escolhido apresenta aspectos dos modelos viscolásticos (Maxwell, Kelvin-Voight e Sólido Linear Padrão) mais utilizados, e cada um deles é descrito pelo modelo de Burger (04 parâmetros) sob certas condições do meio (valores de densidades e viscosidades). Por apresentar essas características, por exemplo: apresenta os aspectos de fluência dos modelos de Maxwell e de Voight, julgamos que o modelo proposto deve recuperar informações melhores do imageamento de subsuperfície. Num primeiro momento utilizaremos dados sinteticos para testar o modelo criado e depois verificaremos a eficácia do mesmo com dados reais. $O$ modelo de 04 parâmetros em conjunto com teorias do fator da qualidade e memória evanescente, apresenta-se como um possível processo de modelagem mais robusto.

\section{Metodologia/ Problema Investigado}

A anelasticidade depende, geralmente, de um grande numero de mecanismos físicos, os quais podem ser modelados por diferentes teorias microestruturais. As propriedades físicas do meio real podem ser representadas por modelos mecânicos. Tais modelos mecânicos são representados por molas (desprezando-se o peso nenhum efeito inercial estará presente, representam o sólido elástico) e amortecedores (Pistão em um cilindro preenchido com um fluido viscoso, representando a parte viscoelástica do sistema e relacionada como um dos mecanismos de perda). Esses modelos são utilizados para modelar as propriedades físicas do meio real e conforme o modelo adotado, todo um processo de modelagem do meio é desenvolvido. Os exemplos abaixo descrevem alguns desses modelos.

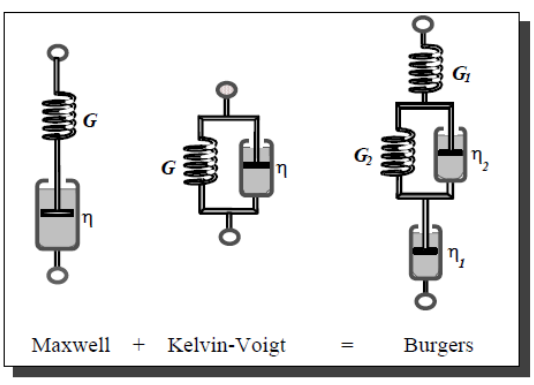

Figura 1 - Modelo de Maxwell, Kelvin-Voight e o Modelo Proposto.

Cada modelo tem seu fator de qualidade e o mesmo é implementado no processo de modelagem.

A teoria da mecânica do contínuo, a simulação numérica e a propagação de ondas são o eixo principal do desenvolvimento do projeto. O método de diferenças finitas é utilizado na implementação dos algoritmos de representação do modelo. $O$ modelo inicial para teste foi o de Maxwell (onda P) e foi implementado como se segue:

- Modelo Viscoelástico de Maxwell adotado para a construção do processo de modelagem do meio.

- a implementação do algoritmo de diferença finita de marcha no tempo ( equação do movimento em 2D);

- a implementação numérica utilizada é a de diferença finitas com peso ( no espaço com aproximação de quarta ordem e segunda ordem no tempo).

\section{Resultados}

Os testes de implementação de modelagem numérica e algoritmo mostraram-se satisfatórios e apresentamos alguns resultados obtidos com 0 algoritmo implementado. Como resultados preliminares temos algumas figuras geradas pelo algoritmo de teste que utilizou o Modelo de Maxwell.

Apresentaremos alguns snapshots e sismogramas em tempo. O modelo geológico usado foi o mais simples possível (modelo de duas camadas). O modelo foi discretizado usando uma malha quadrada em $d x=5$ e $d z=5$. Apresentamos alguns sismogramas que levam em consideração a variação de parâmetros tais como: viscosidade e seus respectivos $\mathrm{Q}$ do fator da qualidade e seus efeitos de atenuação. 


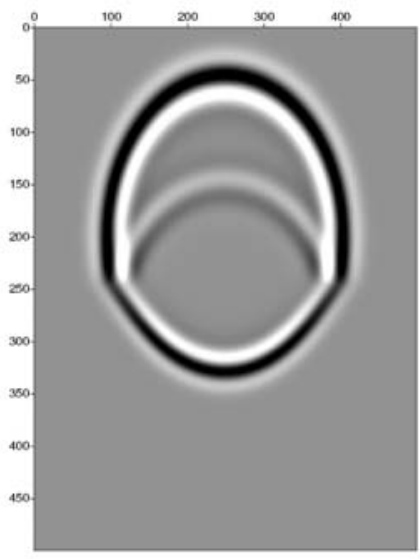

Figura 2 - Snapshot do campo de onda P no instante $\mathrm{t}=1,4 \mathrm{~s}$.

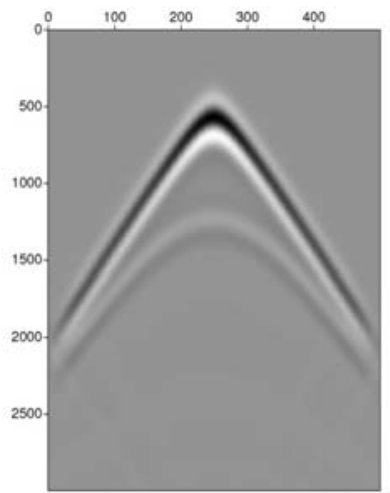

Figura 3 - Sismograma no tempo $t=3,0$ s.

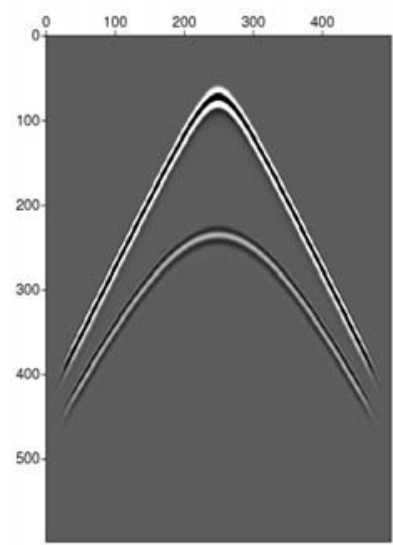

Figura 4 - Sismograma no tempo $t=0,6 s$, frequência da fonte $50 \mathrm{hz}$ e fator de atenuação $\mathrm{Q}=1000$.

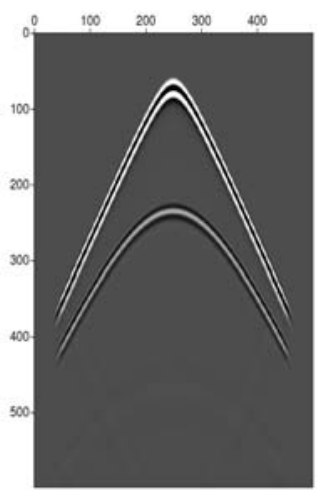

Figura 5 - Sismograma no tempo $t=0,6 s$, frequência da fonte $50 \mathrm{hz}$ e fator de atenuação Q=200.

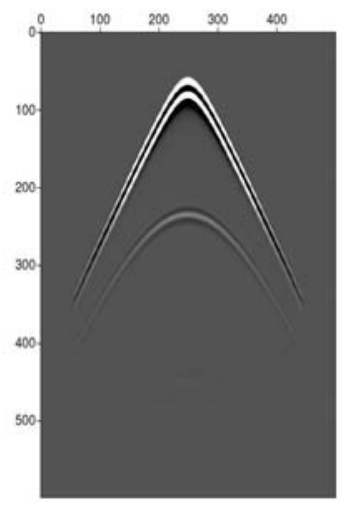

Figura 6 - Sismograma no tempo $t=0,6 s$, frequência da fonte $50 \mathrm{hz}$ e fator de atenuação $\mathrm{Q}=10$

\section{Discussão e Conclusões}

Ao longo da pesquisa temos verificado que poderá haver uma maior aplicação de modelos viscoelásticos na industria petrolífera pois o mesmo leva em consideração a atenuação e dessa forma recuperando informações mais realísticas do meio. No caso de nossa pesquisa, o modelo de Burger incorpora informações de dois modelos ao mesmo tempo (Maxwell e Kelvin-Voight) e dessa forma tem uma característica mais realista de modelagem do meio. Os resultados obtidos recuperam essa informação de atenuação do meio e dessa forma gera sismogramas sintéticos que tem uma melhor exatidão. Esperamos implementar o modelo de Burger e 
conseguir resultados muito promissores e dar nossa contribuição a pesquisa em geofísica e também para área da modelagem.

\section{Agradecimentos}

Agradeço a Capes pela bolsa de estudos, pois sem ela não seria possível o meu trabalho e ao CPGG pelo apoio a mim dispensado.

\section{Referências}

J. M. Carcione, 2001. Theory and numerical simulation of wave propagation in anisotropic, anelastic and porous media. Pergamon Press.

H. Emmerich and M. Korn, 1987. Incorporation of attenuation into time-domain computations of seismic wave fields. Geophysical, 52: 1252-1264.

Carcione J. M., D. Kosloff, R. Kosloff, 1988. Wave propagation simulation in a linear viscoelastic medium. Geophysical Journal, 95: 597-611.

Sun Cheng-yu, YIN Xing-yao, 2007. Construction of constant-Q viscoelastic model with three parameters.

Acta Seismologica Sinica, 20: 370-380. 